\title{
ON THE POWER OF A PRIME DIVIDING THE ORDER OF A GROUP OF AUTOMORPHISMS ${ }^{1}$
}

\author{
J. E. ADNEY
}

1. Introduction and notation. A natural question that arises concerning a finite group $G$ and its group of automorphisms $A(G)$ is the relationship between their orders. P. Hall and G. Birkhoff [1] have obtained an upper bound on the order of $A(G)$ in terms of the order of $G$.

In this paper we shall be concerned with the power of the prime $p$ that divides the order of $A(G)$ for those primes $p$ that divide the order of $G$. For abelian groups Hilton [2] proved that if $p^{n}$ divides the order of $G$, then $p^{n-1}(p-1)$ divides the order of $A(G)$. In the general case Herstein and Adney [3] proved that if $p^{2}$ divides the order of $G$, then $p$ divides the order of $A(G)$. Scott [4] showed that if $p^{3}$ divides the order of $G$, then $p^{2}$ divides the order of $A(G)$.

The main theorem proved in this paper is as follows:

TheOREM. Let $G$ be a group of order $p^{n} g$ where $(p, g)=1, p$ a prime, and let $P$ denote a $p$-Sylow subgroup belonging to $G$. If $P$ is abelian, then at least $p^{n-1}$ divides the order of $A(G)$.

A counterexample to the general conjecture that if $p^{n}$ is the highest power of $p$ that divides the order of $G$ then $p^{n-1}$ divides the order of $A(G)$ is the following: Let $G$ be the group of all quadratic nonsingular matrices with elements from the Galois field with 19 elements then ${ }^{2}$ $[G: 1]=\left(19^{2}-1\right)\left(19^{2}-19\right)$ and $[A(G): 1]=o(G) / 3^{2}$.

Notation. In this paper we shall write group for finite group.

If $G$ is a group, we denote by:

$Z(G)$ the center of $G$,

$G^{\prime}$ the commutator subgroup of $G$,

$A(G)$ the group of automorphisms of $G$,

$I(G)$ the group of inner automorphisms of $G$ and $[G: 1]$ the order of $G$.

If $H$ is a subgroup of $G$, we denote by $V_{G \rightarrow H}(G)$ the transferred group of $G$ to $H[5]$.

Presented to the Society, December 27, 1954; received by the editors May 16, 1955 and, in revised form, August 20, 1956.

1 The following comprises part of a $\mathrm{Ph} . \mathrm{D}$. thesis written under the direction of Professor Marshall Hall and accepted by the Ohio State University. I wish, at the outset, to acknowledge my deep indebtedness to my former teacher.

2 The author is indebted to the referee for this counterexample as well as the form of Theorem 2.2. 
2. Preliminaries. We use the following theorems in the proof of the main theorem of this paper.

THEOREM 2.1. If the finite group $G$ has an abelian $p$-Sylow subgroup $P$, then we have

$$
G=G_{1} \cdot P_{1}, \quad G_{1} \cap P_{1}=1
$$

where $G_{1}$ is the kernel of the transfer of $G$ into $P$ and $P_{1}$ is the image group of $G$ transferred into $P$. Moreover $P_{1}$ contains $P_{2}=P \cap Z(G)$.

PROOF. This theorem is an immediate corollary of [5, pp. 142$143]$.

THEOREM 2.2. Let $G$ be a group, $N$ a normal subgroup, and $R a$ representative subgroup of $G$ modulo $N$. All automorphisms $\alpha$ of $R$ that satisfy the condition

$$
x^{-1} \alpha(x) \in Z(G) \cap R, \quad x \in R
$$

form a group $A_{1}=A(G, N, R)$ of automorphisms of $R$ that is mapped isomorphically onto a group $\bar{A}_{1}$ of central automorphisms of $G$ by the correspondence: $\alpha \rightarrow \bar{\alpha}$ where $\bar{\alpha}(n r)=n \alpha(r) n \in N, r \in R$, and $A_{1}$ is obtained from $\bar{A}_{1}$ by restriction to $R$. If $R$ is abelian then $\bar{\alpha}$ is inner only if $\alpha$ is the identity.

Proof. Clearly the $\alpha$ 's form a group. By direct verification it follows that $\bar{\alpha}$ is an automorphism of $G$. Moreover from the definition of $\bar{\alpha}$ and the fact that $\alpha$ is an automorphism the correspondence $\alpha \rightarrow \bar{\alpha}$ is $1-1$ and preserves products. This isomorphism together with $\bar{\alpha}(1 \cdot r)=\alpha(r)$ shows that $\bar{A}_{1}$ restricted to $R$ gives $A_{1}$.

Now let $R$ be abelian. If $\bar{\alpha}$ is an inner automorphism we have

$$
\bar{\alpha}(n r)=n r z=g n r g^{-1}=n_{1} r_{1} n r r_{1}^{-1} n_{1}^{-1} \text { where } g=n_{1} r_{1} .
$$

But then

$$
z=n^{-1} n_{1} r_{1} n r_{1}^{-1} r n_{1}^{-1} r^{-1} \in N \text { since } N \text { is normal and is abelian. }
$$

Hence $z=1$ and $\alpha(r)=r$.

3. Construction of central automorphisms. In the remainder of the paper it is assumed (i) that $G$ is a group of order $p^{n} g$ where $p$ is a prime, $(g, p)=1$, and (ii) that a $p$-Sylow subgroup $P$ of $G$ is abelian. Let $P_{1}=V_{G \rightarrow P}(G),[P: 1]=p^{n}=p^{r+s},\left[P_{1}: 1\right]=p^{r},\left[P_{2}: 1\right]=p^{s}$ and let $G_{1}$ denote the kernel of the transfer of $G$ into $P$. If $p^{m}$ is the highest power of $p$ dividing $\left[A_{1}: 1\right]$ where $A_{1}=A\left(G, G_{1}, P_{1}\right)$ the two previous results show that $p^{m} \mid[A(G): I(G)]$. Since $p^{r} \mid[I(G): 1]$ it follows that 
$p^{m+r} \mid[A(G): 1]$. It is to be shown that $m+r \geqq n-1=r+s-1$ or $m \geqq s-1$.

The main theorem has now been reduced to a consideration of the automorphisms $\alpha$ of an abelian $p$-group $P_{1}$, with a subgroup $P_{2}$ of order $p^{s}$, such that $x^{-1} \alpha(x) \in P_{2}$, for all $x \in P_{1}$.

We proceed to construct the required automorphisms of $P_{1}$ with the repeated application of the following:

REMARK. An element of highest order in an abelian p-group may be chosen as a basis element [6].

Consider first $P_{2} \subset P_{1}$. Let $\left\{a_{1}, \cdots, a_{k}\right\}$ be a basis for $P_{1}$ with $a_{i}$ of order $p^{m_{i}}$ and let $a_{1}$ be an element of highest order in $P_{1}$. If $P_{2}$ does not contain an element of highest order, then $a_{1} \notin P_{2}$ and we define

$$
\begin{array}{rlrl}
\alpha\left(a_{1}\right) & =a_{1} \cdot b & b \in P_{2}, \\
\alpha\left(a_{i}\right) & =a_{i} & i \neq 1 .
\end{array}
$$

We assert $\alpha$ is an automorphism of $P$. Suppose

$$
\left(a_{1} b\right)^{x_{1}} a_{2}^{x_{2}} \cdots a_{k}^{x_{k}}=1 \text { with not all } x_{i}=0 .
$$

In particular $x_{1} \neq 0$ for otherwise there would be a dependence among $\left\{a_{2}, a_{3}, \cdots, a_{k}\right\}$. Since

$$
b \in P_{2} \subset P_{1}
$$

we can write

$$
b=a_{1}^{y_{1}} a_{2}^{y_{2}} \ldots a_{k}^{y_{k}}
$$

with $y_{1}$ a multiple of $p$, since $b$ is of lower order than $a_{1}$. Now we obtain

$$
a_{1}^{x_{1}+x_{1} y_{1}} a_{2}^{x_{2}+x_{1} y_{2}} \cdots a_{k}^{x_{k}+x_{1} y_{k}}=1
$$

and this implies

$$
x_{i}+x_{1} y_{i} \equiv 0\left(\bmod p^{m_{i}}\right) .
$$

But

$$
\begin{gathered}
x_{1}+x_{1} y_{1} \equiv x_{1}\left(1+y_{1}\right) \equiv 0\left(\bmod p^{m_{1}}\right), \\
\left(1+y_{1}, p\right)=1
\end{gathered}
$$

hence $x_{1}=p^{m 1}$. Thus

$$
a_{2}^{x_{2}} \cdots a_{k}^{x_{k}}=1
$$

and hence 


$$
x_{2}=x_{3}=\cdots=x_{k}=0
$$

so that

$$
\left\{a_{1} b, a_{2}, \cdots, a_{k}\right\} \text { is a basis for } P .
$$

By direct calculation one can show that for $a, a^{\prime} \in P_{1}$

$$
\alpha\left(a a^{\prime}\right)=\alpha(a) \alpha\left(a^{\prime}\right) .
$$

The product of any two such automorphisms is again an automorphism of the same type. For let

$$
\begin{array}{lrl}
\beta\left(a_{1}\right) & =a_{1} \cdot b^{\prime}, & b^{\prime} \in P_{2}, \\
\beta\left(a_{i}\right)=a_{i}, & i \neq 1,
\end{array}
$$

then

$$
\alpha \beta\left(a_{1}\right)=\alpha\left(a_{1} b^{\prime}\right)=a_{1} b \alpha\left(b^{\prime}\right)=a_{1} b
$$

where

$$
\bar{b}=b \alpha\left(b^{\prime}\right) \in P_{2} .
$$

Therefore the set of automorphisms obtained as $b$ runs over $P_{2}$ form a group. By direct calculation $\alpha$ has the same order as the corresponding $b \in P_{2}$ and we have at least $p^{8}$ automorphisms.

If, on the other hand, $P_{2}$ contains an element of highest order in $P_{1}$, say $a_{1}$, we choose a basis for

$$
P_{1}\left\{a_{1}, a_{2}, \cdots, a_{k}\right\} \text {. }
$$

We can write $P_{1}=\left\{a_{1}\right\} \times H_{1}$ where $\left\{a_{1}\right\}$ denotes the cyclic group of order $p^{m_{1}}$ generated by $a_{1}$. By a proper choice of a basis for $P_{2}$ we can write $P_{2}=\left\{a_{1}\right\} \times K_{1}$ such that $K_{1} \subset H_{1}$.

Suppose now that $a_{2} \in H_{1}$ is an element of highest order in $H_{1}$. Also suppose that $K_{1}$ does not contain an element of highest order of $H_{1}$. Then since $a_{2} \notin K_{1}, a_{2}$ of order $p^{m_{2}}$, we define

$$
\begin{array}{llrl}
\alpha\left(a_{2}\right)=a_{2} \cdot b, & & b \in K_{1}, \\
\alpha\left(a_{i}\right) & =a_{i}, & & i \neq 1,2
\end{array}
$$

and $\alpha\left(a_{1}\right)$ any of the $p^{m_{1}-1}(p-1)$ automorphisms of $\left\{a_{1}\right\}$. Apply the previous argument to $H_{1}$ and $K_{1}$. Since the direct product of automorphisms of the direct factors of a group is a subgroup of the automorphism group of the group itself, we have, therefore, $p^{o-1}$ automorphisms. We can construct an additional automorphism of order $p$ of $P_{1}$ by defining: 


$$
\begin{aligned}
& \beta\left(a_{2}\right)=a_{2} \cdot a_{1}^{p_{1}^{m_{1}-1}}, \\
& \beta\left(a_{i}\right)=a_{i},
\end{aligned}
$$

$\beta$ is an automorphism of $P_{1}$. Suppose

$$
a_{1}^{x_{1}}\left(a_{2} \cdot a_{1}^{p^{m_{1}-1}}\right)^{x_{2}} a_{3}^{x_{3}} \cdots a_{k}^{x_{k}}=1
$$

with not all $x_{i}=0$. In particular, $x_{2} \neq 0$, for otherwise we would have a dependence among

$$
a_{1}, a_{3}, \cdots, a_{k}
$$

Simplifying we get

$$
a_{1}^{x_{1}+x_{2} p^{m_{1}-1}} a_{2}^{x_{2}} \cdots a_{k}^{x_{k}}=1
$$

but this implies

$$
x_{1}+x_{2} p^{m_{1}-1} \equiv 0\left(\bmod p^{m_{1}}\right), \quad x_{i} \equiv 0\left(\bmod p^{m_{i}}\right), \quad i=2, \cdots, k .
$$

However, since $x_{2} \neq 0$, we have a contradiction. Also $\beta$ is of order $p$ since $\left\{a_{1}\right\}$ is fixed elementwise by $\beta$ and $a_{1}^{p^{m 1-1}}$ is of order $p$. $\beta$ involves both direct factors, hence it is not one of the previously constructed automorphisms whence $p^{s}$ automorphisms have been constructed. However, if $a_{2} \in K_{1}$, to $P_{1}$ and $P_{2}$, respectively. We have $H_{2} \subset H_{1}$ and $K_{2} \subset K_{1}$ so that

$$
\begin{aligned}
& H_{1}=\left\{a_{2}\right\} \times H_{2}, \\
& K_{1}=\left\{a_{2}\right\} \times K_{2}
\end{aligned}
$$

where $K_{2} \subset H_{2}$. As before, we construct the automorphisms of $H_{1}$ (in the role of $P_{1}$ ) and since $H_{1}$ is a direct factor of $P_{1}$ the automorphisms will be automorphisms of $P_{1}$, using $H_{1}$ exclusively. In fact, since

$$
P_{1}=\left\{a_{1}\right\} \times H_{1} \text { we have } A\left(P_{1}\right) \supset A\left(\left\{a_{1}\right\}\right) \times A\left(H_{1}\right) \text {. }
$$

Also since we have $p^{m_{1}}$ automorphisms using $a_{1}$ exclusively, we have the required $p^{s}$ automorphisms.

Suppose we have

$$
\begin{aligned}
& P_{1}=\left\{a_{1}\right\} \times\left\{a_{2}\right\} \times \cdots \times\left\{a_{i}\right\} \times H_{i}, \\
& P_{2}=\left\{a_{1}\right\} \times\left\{a_{2}\right\} \times \cdots \times\left\{a_{i}\right\} \times K_{i}
\end{aligned}
$$

where $K_{i} \subset H_{i}$ and

$$
\begin{aligned}
& P_{1} \supset H_{1} \supset \cdots \supset H_{i}, \\
& P_{2} \supset K_{2} \supset \cdots \supset K_{i}
\end{aligned}
$$


Also $a_{j}$ is an element of highest order in

$$
H_{i}, K_{j}, \quad \text { for } j=1,2, \cdots, i-1 .
$$

Now either $K_{i}$ contains an element of highest order of $H_{i}$ or $K_{i}$ does not contain an element of highest order of $H_{i}$. In the latter case let $a_{i+1}$ be an element of highest order in $H_{i}$; hence, $a_{i+1} \notin K_{i}$ and we define

$$
\begin{aligned}
\alpha\left(a_{i+1}\right) & =a_{i+1} b, & b \in K_{i}, \\
\alpha\left(a_{t}\right) & =a_{t}, & t=i+2, \cdots, k
\end{aligned}
$$

and $\alpha\left(a_{t}\right)$ is any of the $p^{m_{t}-1}$ automorphisms of the cyclic group generated by $a_{t}(t=1,2, \cdots, i)$. For each $a$ a further automorphism may be constructed as follows: Define

$$
\begin{array}{rlrl}
\gamma\left(a_{r}\right) & =a_{r} \cdot a_{1}^{p^{m-1}-1}, & r=1,2, \cdots, i+1, \\
\gamma\left(a_{t}\right)=a_{t}, & \text { for } t \neq r .
\end{array}
$$

Hence we have the required $p^{s}$ automorphisms by application of the previous arguments.

If, however, $K_{i}$ contains an element of highest order of $H_{i}$, say $a_{i+1}$, then, as before, we have

$$
\begin{aligned}
& P_{1}=\left\{a_{1}\right\} \times \cdots \times\left\{a_{i+1}\right\} \times H_{i+1}, \\
& P_{2}=\left\{a_{1}\right\} \times \cdots \times\left\{a_{i+1}\right\} \times K_{i+1}
\end{aligned}
$$

where $K_{i+1} \subset H_{i+1}$. But $P_{2} \subset P_{1}$; hence we have for some $i \leqq k$ the first case of $H_{i+1} \neq 1$ and $K_{i+1}=1$. Finally, if

$$
P_{2}=P_{1}=P \cap Z(G) \text {, }
$$

then

$$
G=G_{1} \times P_{1} \text { and } A(G) \supseteq A\left(G_{1}\right) \times A\left(P_{1}\right)
$$

Now

$$
p^{s-1} \mid\left[A\left(P_{1}\right): 1\right] \text { by }[1] \text { and } p^{r} \mid[(G): 1] \text {. }
$$

Since $I(G)$ fixes $P_{1}$ elementwise, we have $p^{r+s-1}=p^{n-1} \mid[A(G): 1]$.

It should be noted that the results of this section include Theorem 1 of Scott [4].

\section{BIBLIOGRAPHY}

1. G. Birkhoff and P. Hall, On the order of groups of automorphisms, Trans. Amer. Math. Soc. vol. 39 (1936) pp. 498-499.

2. H. Hilton, On the order of the group of automorphisms of an Abelian group, Messenger of Math. (2) vol. 38 (1909) pp. 132-134. 
3. I. N. Herstein and J. E. Adney, A note on the automorphism group of a finite group, Amer. Math. Monthly vol. 59 (1952) pp. 309-310.

4. W. R. Scott, On the order of the automorphism group of a finite group, Proc. Amer. Math. Soc. vol. 5 (1954) pp. 23-24.

5. H. Zassenhaus, The theory of groups (English trans.), New York, 1949.

6. W. Burnside, Theory of groups, Cambridge, 1897.

The Ohio State University

\section{A NOTE ON FINITE UNIONS OF IDEALS AND SUBGROUPS}

NEAL H. MCCOY

1. Introduction. From the theory of ideals in a commutative ring $R$ it follows easily that an ideal $I$ of $R$ is contained in the (settheoretic) union of a finite number of prime ideals $P_{i}(i=1,2, \cdots, n)$ of $R$ if and only if $I$ is contained in some one of the ideals $P_{i}$. A simple direct proof of this will be found in [2, p. 186]. Recently, Behrens $[1$, p. 171] has shown that the same result holds for the case in which neither commutativity nor associativity is assumed in $R$.

It is easy to see that if an ideal $I$ of a ring $R$ is contained in the union $A_{1} \cup A_{2}$ of any two ideals $A_{1}$ and $A_{2}$, it must be contained in one of them. For suppose that $I \subseteq A_{1} \cup A_{2}$ and that $I \nsubseteq A_{1}$. Then there exists an element $a_{2}$ of $I \cap A_{2}$ such that $a_{2} \notin A_{1}$. If $x \in I \cap A_{1}$, then $x+a_{2} \notin A_{1}$ and therefore $x+a_{2} \in A_{2}$ and $x \in A_{2}$. That is, $I \cap A_{1} \subseteq A_{2}$ and we have $I \subseteq A_{2}$. As a matter of fact, this result remains valid if $I, A_{1}$, and $A_{2}$ are subgroups of an arbitrary group. These observations were pointed out to me by Bailey Brown who also raised several questions about possible generalizations, some of which are partially answered in this note.

The following simple example, due to R. E. Johnson, shows that the above result about the union of two ideals no longer holds when we pass to the union of three ideals. Let $R$ be the ring whose additive group is the direct sum of two two-element cyclic groups, with every products equal to zero. Thus the elements of $R$ may be written as $(0,0),(0,1),(1,0)$, and $(1,1)$ with componentwise addition modulo 2. Then $A_{1}=\{(0,0),(0,1)\}, A_{2}=\{(0,0),(1,0)\}$, and $A_{3}=\{(0,0)$, $(1,1)\}$ are ideals in $R$, and $R$ is contained in the union of these three

Received by the editors September 30, 1956. 